\title{
DESIGN AND IMPLEMENTATION OF A WIRELESS SENSOR NETWORK SERVICE SYSTEM USING A CC2430 CHIP AND MEMS TECHNOLOGY
}

\author{
Hanzhong Kang \\ Georgia Institute of Technology, North Avenue, Atlanta, GA 30332, USA \\ Email: hanzhonggit@126.com
}

\begin{abstract}
The purpose of this work is to improve the utilization efficiency of WSN (Wireless Sensor Network) through the WSN service system based on MEMS (Micro-Electro-Mechanical System) technology. Here, a WSN service system with a CC2430 chip (integrated with a microcontroller) is innovatively proposed through WSN and SOA (Service-Oriented Architecture), and the optimized genetic algorithm is chosen for node control. Finally, the proposed system is experimentally simulated through MATLAB (Matrix Laboratory). The results show that the proposed system can display the overall serviceability of the network on the webpage. Meanwhile, the proposed system can show the results of temperature data acquisition with the range of 288:321, and the numerical type is the maximum value. The electrical control webpage of the system can manually close the air conditioner and can automatically control the wall lamp. In the case of a node failure, with 150 times iterations, the differences are small of the three tests in the number of nodes, path distance, and maximum distance. When the distance between the test content and the node is less than two meters, the number of received packets and the number of sent packets are the same, and the error number and packet loss rate are 0 . When the distance between the test content and the node is over two meters, with the increase of distance, the number of packets received gradually reduces, and the packet error rate and packet loss rate increase. Consequently, the proposed system is feasible, the expectation on system service efficiency can be met, and it can be applied to the actual WSN service field.
\end{abstract}

Keywords: Wireless Sensor Network; Service Architecture; CC2430 Chip; Microcontroller; Wireless Sensor Network Service System.

\section{Introduction}

WSN (Wireless sensor network) technology appeared in the late 1990s and has been widely used in military departments, academia, and industry. With the rapid development and increasing demand, sensor technology has become an extremely complex system, including CPU (Central Processing Unit), memory, wireless communication, and sensor equipment $[1,2]$. The sensor network is a distributed computer system of sensor nodes that acquire and transmit data, including light, temperature, humidity, simultaneous interpretation information. WSN is a heated topic in IoT (Internet of Things). There is still no large-scale and successful application of sensor networks in the industry field, and it has just been introduced into the market [3]. The sensor network can acquire data accurately. Hence, a real-time detection system and an early warning system can be established through the senor network, and meanwhile, a fast and effective emergency command system can be constructed to help people deal with natural disasters, such as forest fires, tsunami. In the past, a sensor network is a closed network system with specific applications for monitoring environmental data $[4,5]$. Presently, many challenges have been put forward to the research and development of sensor networks: one issue is to quickly and effectively develop, deploy, run, and manage network service resources and abilities. The next problem is to realize the cooperation of application services between intelligent nodes and systems. One other thing is to provide the sensing and control abilities of heterogeneous sensor networks. Lastly, a unified and abstract means should be used by the Internet users and service providers [6].

Global sensor networks have introduced service architecture into the WSN. A leading sensor network system design supports users around the world to easily and uniformly use the sensor equipment and can add sensors to the network system to provide reliable services for other users [7]. Based on the global SOA (Service-Oriented Architecture), sensor 
devices with completely different structures can share the acquired data and calculate the sensor data through the network. With the further development of global sensor networks, many research units or organizations have joined the research on serviceoriented networks. Oswa is a kind of serviceoriented sensor network developed by the NICTA (National Information \& Communication Technology Australia) Laboratory of Melbourne University. The whole architecture consists of three parts: sensor structure layer, service layer, and development and application layer. The underlying layers provide services to connect the source, and the higher layer creates applications and manages sensor networks. Oswa is a comprehensive open platform, which can manage sensor networks in a service-oriented manner, and it is also a new distributed computing platform, such as a grid computing platform [8].

Meanwhile, the sensor networks should not only be application-oriented but also should implement the features, such as low cost, low energy consumption, and unlimited resources of sensor networks, into practical application [9]. Hence, a fresh improved service model can be established. The registration and management technology of sensor networks can provide the services provided by sensor networks to the outside world. The study is much limited in the management of the internal service of sensor networks. Currently, the management of service itself is analyzed, while the service interruption caused by node failure or dormancy is barely considered [10]. Therefore, it is necessary to study the basic principle and technology of internal service management in sensor networks.

To sum up, based on the theoretical analysis of WSN and SOA, an optimized genetic algorithm is proposed for node positioning. Then, a WSN service system is constructed using the CC22430 chip and MEMS (Micro-Electro-Mechanical System) technology. Afterward, the system is simulated and evaluated through algorithm and service environment, and an experimental basis is provided for the practical application of the WSN service system.

\section{Design of Service-oriented MEMS WSN System 2.1 WSN}

WSN [11] is a freely organized network of sensor nodes connected through wireless communication technology. A sensor node includes a data acquisition unit, data transmission unit, data processing unit, and energy supply unit. The data acquisition unit usually acquires and converts information, such as light intensity, atmospheric pressure, and humidity for the monitoring area.
The data transmission unit focuses on wireless communication and information exchange, and sends and receives the acquired data. Data processing units typically handle routing protocols, node management, and location devices. For occupation reduction, the energy supply unit often chooses the micro battery. There are two types of nodes in WSNs: the sink node and the sensor node [12]. The sink node refers to the gateway that can eliminate the error report in the sensor node and judge the event through a fusion data report. The sink node can communicate with the user node through WAN (Wide Area Network) or satellite, and process the acquired data.

Firstly, the composition of WSN is introduced. WSN is composed of nodes, sensor networks, and users [13]. The monitored area should be covered with nodes according to specific requirements. The sensor network is a vital part. The data of all nodes are acquired through a fixed channel, analyzed, and calculated. Then, the analysis results are analyzed of the base station and transmitted to the designated user through satellite communication. This is an overall procedure of wireless sensing, as shown in figure 1.

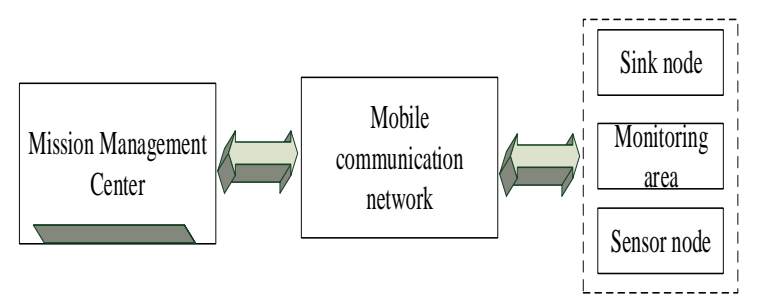

Figure. 1 Architecture of sensor network

The sensor node is the sensing part, and the data sink node is the data export of the sensor network. The last part is the task management center. Many sensor nodes are deployed in or near the monitoring area through broadcast and organized into wireless networks. The user sends out the task of acquiring data, and the node receives the sensor data for acquisition. These data can be transmitted to other nodes through routers or can be comprehensively processed through transmission nodes. Finally, the data arrive at the sink node in the network and are reported to the user. Users can also manage and configure sensor networks according to rules.

Secondly, the hardware components of a sensor node include a sensor module, wireless communication module, processor module, and energy supply module [14]. The sensor module monitors and converts all kinds of environmental data. The processor module is the hub of the node and controls the function of the whole node and the CPU. The wireless communication module can communicate with the nodes in the sensor network. The energy supply module can provide energy for other modules with a micro battery. The sensor node composition is shown in figure 2. 


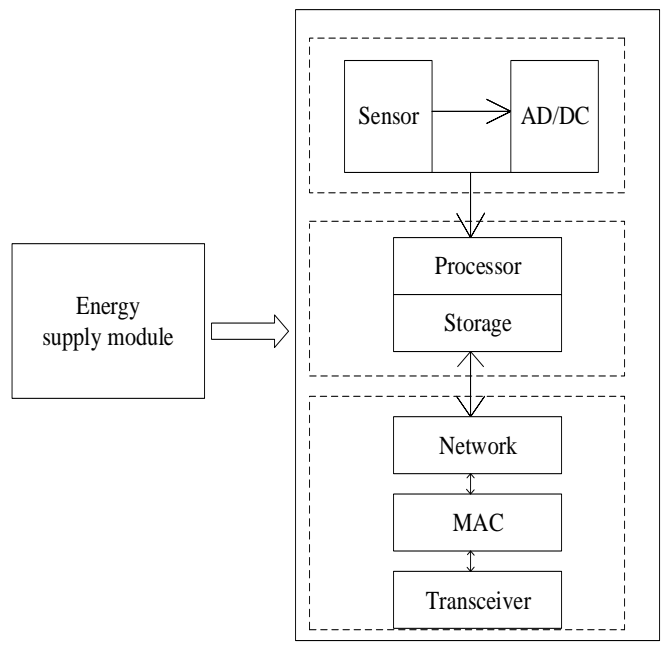

Figure. 2 Components of sensor node

Many wireless network protocols are used in WSN, including the five-layer protocol structure which includes the application layer, transport layer, network layer, data link layer, and physical layer [15]. The energy management platform, task management platform and mobile management platform are included in the network protocols for reasonable control and enhanced management of sensor networks. The management-side protocol stack can improve the efficiency of sensor nodes and reduce energy consumption. The functions of the five-layer protocol are shown in table 1.

Table 1 Protocol of wireless network

\begin{tabular}{|l|l|}
\hline Hierarchy type & Function \\
\hline Physical layer & $\begin{array}{l}\text { Physical layer can modulate and transceiver wireless signals } \\
\text { and can select the wireless channel to transmit a bit } \\
\text { transparently. }\end{array}$ \\
\hline Datalink layer & $\begin{array}{l}\text { The data link layer can provide post-detection and error } \\
\text { control functions and can encapsulate the network layer data } \\
\text { into the device. }\end{array}$ \\
\hline Network layer & $\begin{array}{l}\text { Network layer can select the route of data transmission and } \\
\text { determine the transmission path }\end{array}$ \\
\hline Enansport layer & $\begin{array}{l}\text { Transport layer is crucial for communication quality and can } \\
\text { control data flow in a network. }\end{array}$ \\
\hline Application layer & It can fully control the energy consumption in the node \\
\hline
\end{tabular}

Finally, the features of WSN are explained. The first feature is free organization. The construction of WSN is not restricted by any external conditions. A fully functional WSN can be established whenever and wherever. The WSN can be maintained and managed through the network. The second feature is the uncertainty of network topology. The topology of WSN is variable on the network level. For example, the sensor nodes in the network topology can increase or decrease at any time, and the network topology graph can be separated or merged at any time. The third is not centralized control. Although WSN control both base stations and sensor nodes, the control mode between each sensor node is still decentralized. Routing and host functions are realized through network terminals. Each host runs independently without interference. Hence, the WSN has a high intensity and is not likely to be destroyed.
The fourth feature is not safe. WSN transmits information through the wireless network, so sensor nodes are vulnerable to external invasion during data transmission, resulting in information leakage and damage to WSNs. Most WSN nodes are exposed, reducing the security [16].

\subsection{SOA (Service Oriented Architecture)}

SOA [17] is a component model that splits different functional units, called services, of an application and connects them through well-defined interfaces and protocols. Interfaces are defined neutrally and should be independent of hardware platforms, operating systems, and programming languages.

Thus, the built-in services in various systems can interact in a unified and common way. 
The development of web service technology [18] has rendered the interaction between information systems more effective. SOA modeling method can establish a loose combination between systems and can realize system integration and cooperation. The essence of SOA and web service technology is to complete work through effective communication and technical cooperation. The typical SOA architecture is shown in figure 3.

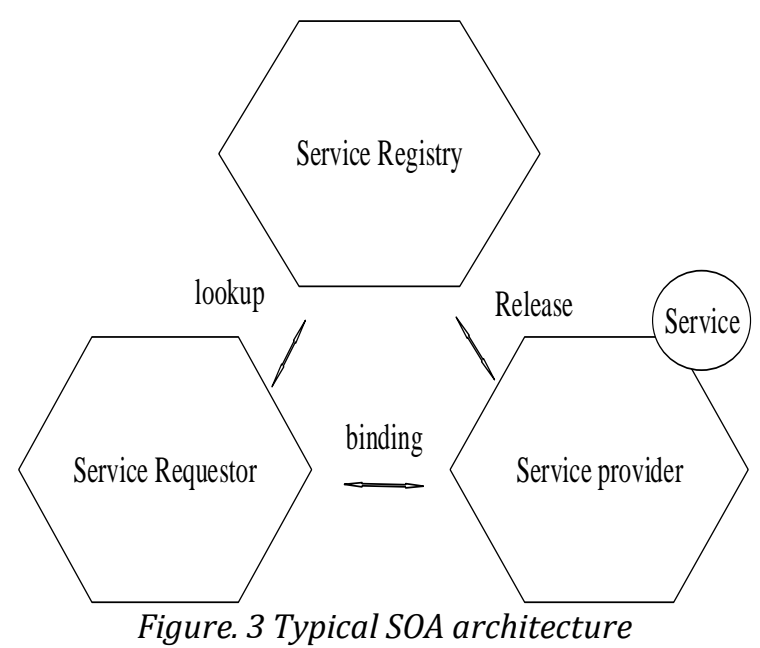

There are three kinds of operations in SOA: the first operation is to release, provide convenient access to services, and release the service description to the registry for lookup and binding.

The release is a service registration and service registration behavior. As a communication bridge between service registries and service providers, the service registry receives the service description from the service provider and informs service requestors of the details of the description. Released operations are based on specific requirements of the service registry. There are two kinds of release operations. One is to transmit the service description to the directory of the server, and the other is to establish a separate management module to manage the service description. The second operation is lookup.

The service requestor initiates the lookup operation and lookup the service registration center. The lookup operation describes the matching conditions according to the service released by the service provider and returns the service results. The third operation is binding and using. After the lookup operation, the service requester can use the query services and use the method specified in the lookup service description. Binding operation is the system configuration behavior of the service provider to the looked-up service and set the parameters specified by the lookup service. Once the binding operation is completed, services can be invoked remotely.

\subsection{Design of WSN Service System based on MEMS Technology}

Firstly, fully inclusive information services from sensor nodes to end-users is the developmental direction of IoT services in the future [19]. Thus, it is expected that a service delivery system can be built, which contains the sensor network, enterprise network, the Internet, and the distributed service delivery system. Sensor nodes can provide services to sink nodes, so how to provide services to Internet users becomes the key to establishing the service delivery system. Consequently, two architectures are suggested: Internet-based architecture and sensorbased architecture [20]. The CC2430 [21] is utilized to design a WSN service system for MCU (Micro Controller Unit) sensors. CC2430 is a real SOC (System on Chip) CMOS (Complex Metal Oxide Semiconductor) solution, which can improve the performance and meet the requirements of $2.4 \mathrm{GHz}$ ISM (International Safety Management) based on ZigBee. It is low in cost and energy consumption. Besides, it integrates a $2.4 \mathrm{GHz}$ DSSS (DirectSequencing Spread-Spectrum) RF transceiver core, the industrial-grade compact 8051 microcontrollers, 8kbyte RAM (Random Access Memory), and powerful peripheral modules. Meanwhile, it has three different versions that optimize the integration complexity and cost utilizing three different flash memory of 32,64 , and $128 \mathrm{kbyte}$. The designed WSN service system is shown in figure 4 .

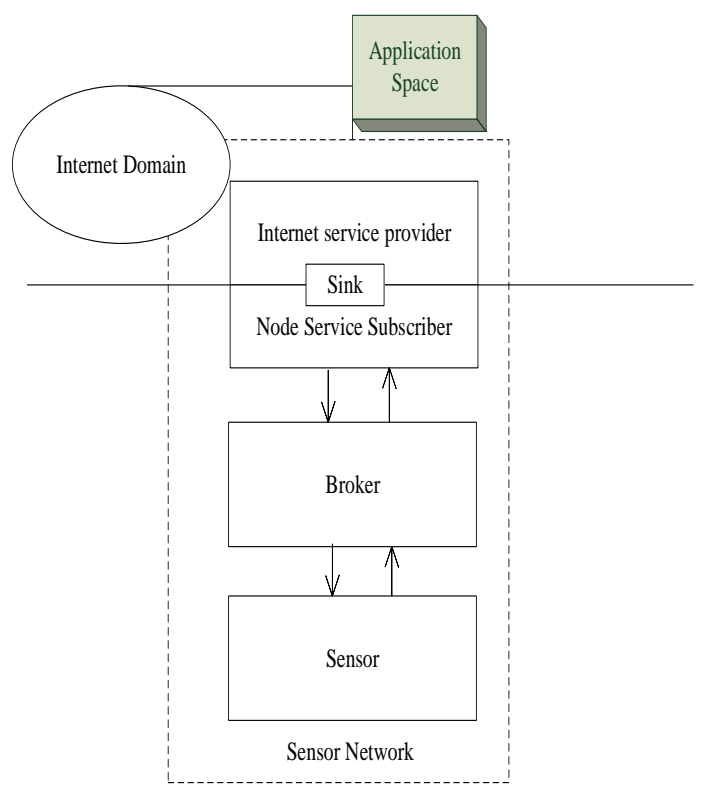

Figure. 4 WSN based on SOA

Secondly, sensor nodes can provide basic services, such as temperature, humidity, pressure, sound, image, video, and electrical equipment operation [22]. 
According to the service content, the sensor network nodes can be divided into two types: acquisition sensor nodes and electrical control sensor nodes. The two nodes are composed of two modules, and the wireless transmission module can construct a wireless network and data transmission. The other module can acquire the environmental data of sensor nodes with the sensor backplane, while the control sensor node backplane is composed of switches. Sensor nodes are also the service providers of the sensor network services. Sensor networks are dynamically and randomly deployed networks, so are often faced with changes, such as dormancy, failure, deletion, or insertion of new nodes [23]. For node insertion, the serviceability can be extended through the service registry at its entry node. To delete the failure node, the fault dynamic discovery and adaptive fault tolerance mechanism based on passive diagnosis are adopted for fast fault detection and fault tolerance, and the registry is altered. Most nodes in WSN use wireless communication protocol, namely ZigBee protocol [24]. For low energy consumption, a simplified design, and a secure protocol, the secret pipeline management mechanism can search the protocol.

Lastly, the node positioning algorithm is introduced. Here, the optimized genetic algorithm [25] can position the nodes. First, the initial value of the population in the monitoring area is represented as the node number, and the fitness value of each node is calculated. Let the initial value of the population $N$ be 150 and the fitness of individual $x$ be $f(x)$, and then the following equation can be obtained.

$$
P=\frac{f(x)}{\sum_{x=1}^{N} f(x)}
$$

After the fitness calculation, the minimum value is eliminated, and the optimal individual is positioned. Then, the coefficient of variation is randomly selected in the interval $[0,1]$, and the threshold is set as the number of iterations. When the optimal solution is obtained, the iteration is stopped.

\subsection{Parameters Setting of the Simulation Experiment}

(1) The software and hardware environment of the system is shown in table 2 .

Table 2 The software and hardware environment

\begin{tabular}{cc}
\hline Hardware & Software \\
\hline CC2430 chip and ARM10 embedded development board & ARMGCC cross compiler tool \\
Average PC & Tomcat 6.0 \\
\hline
\end{tabular}

The system is developed with the $\mathrm{C}$ language under the Linux system, and the gateway communication model can simultaneously communicate with the server and the sensor network. The default password of the node is setup through DEFAULT_JCEY.

(2) The hotel room is chosen for the system simulation base. The user selects the range of interest to obtain the environment data, and the sensor network sends the service request information. The sensor node can be automatically configured for environment data collection according to user acquisition mode and range of the sensor, and the environment data are sent to the server in real-time.

(3) MATLAB can simulate the algorithm, and Origin 2018 64Bit can visually analyze the data.

\section{Test Performance of Intelligent WSN Service System}

\subsection{Test Results of System Service Processing}

The temperature data of the room is analyzed after the system service is registered. The results are shown in figure $5 \mathrm{AB}$.

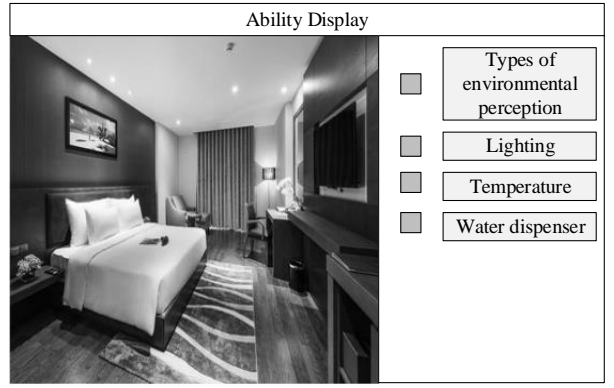

A

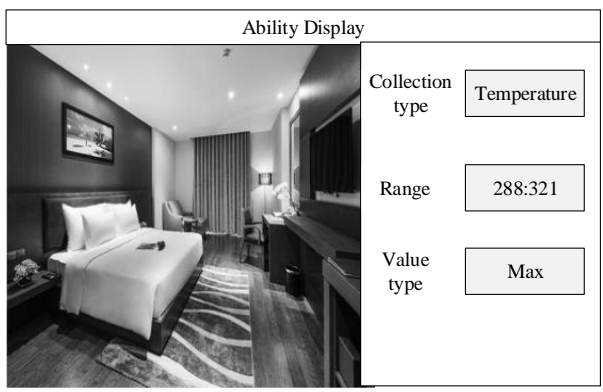

Figure. 5 Test results of system service processing (A: serviceability acquisition results $B$ : environmental data acquisition results) 
Figure A shows that the system can display the overall serviceability of the network on the webpage. Meanwhile, the system can show the acquisition results of temperature data from 288:321, and the numerical type is the maximum value.

A service can be registered after a correct node configuration, and then is reported to the agents with its service abilities when entering the network. The compressed serviceability data of the sensor network is concentrated in the sink node through a bottom-up report. Meanwhile, the overall serviceability can be obtained through the serviceability transmission from the sink node to the server. Afterward, the selected control points can be controlled individually through a webpage displayed with service abilities.
Besides, the user-interested environment data can be selected and sent to the underlying gateway via a Socket communication. Then, the service request information is distributed to the following sensor network via the Sink node

\subsection{Manual and Automatic Control Results of Electrical Appliances in the Room}

The electrical appliances can be opened and closed on the ability display webpage of the system, and the scope of the room can be selected for automatic control. Here, the state of the wall lamp is controlled, as shown in figure $6 \mathrm{AB}$.

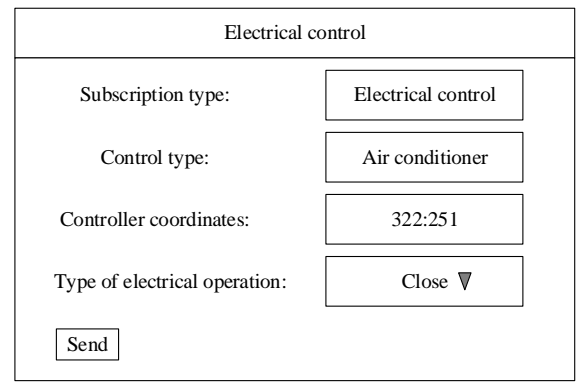

A

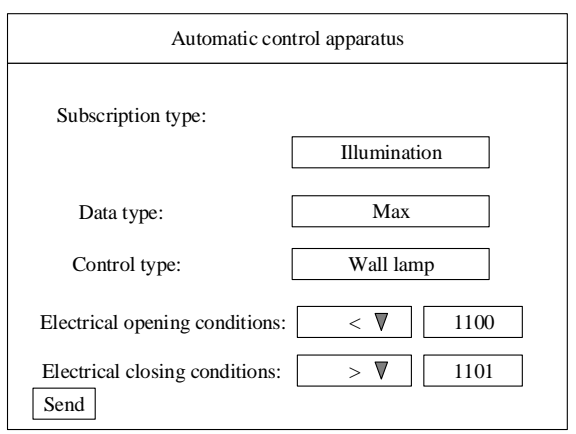

Figure. 6 Manual and automatic control results of electrical appliances in the room (A: manual control B: automatic control)

Figure A shows that on the electrical control webpage of the system, users can close the air conditioner. Figure B indicates that the wall lamp can be automatically controlled within the lighted area of the room. The wall lamp can be automatic controlled as long as the opening conditions are set.

\subsection{Performance of Optimized Genetic Algorithm on the System}

The self-organizing ability of the algorithm is verified. The specific effect is studied of the proposed algorithm on room environmental monitoring in the case of node failure in the simulation system. Three experiments are conducted. The results are shown in figure 7 .

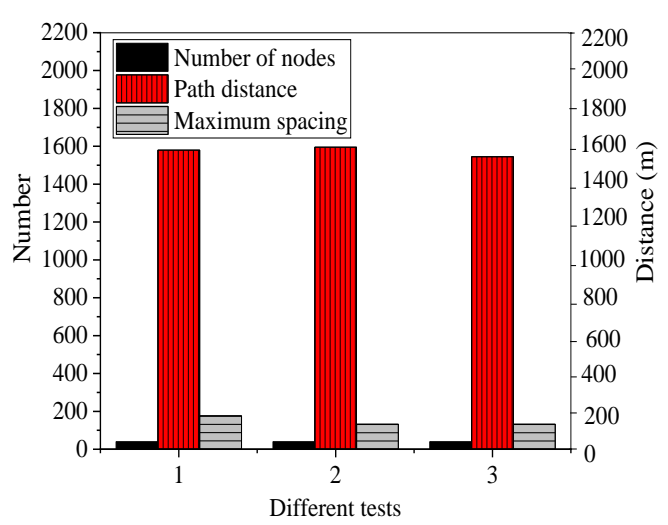

Figure. 7 Performance of optimized genetic algorithm in the system 
Figure 7 shows that even if node failure occurs, data still can be monitored and transmitted smoothly between the sensors. With 150 times iterations, the differences in the number of nodes, path distance, and maximum distance are small of the three test results. It can be implied that if the distance between the adjacent two nodes is within the threshold the overall communication can be improved of the line.

\subsection{System Test Results}

Visual analysis of the test results of the system is shown in figure $8 \mathrm{AB}$.
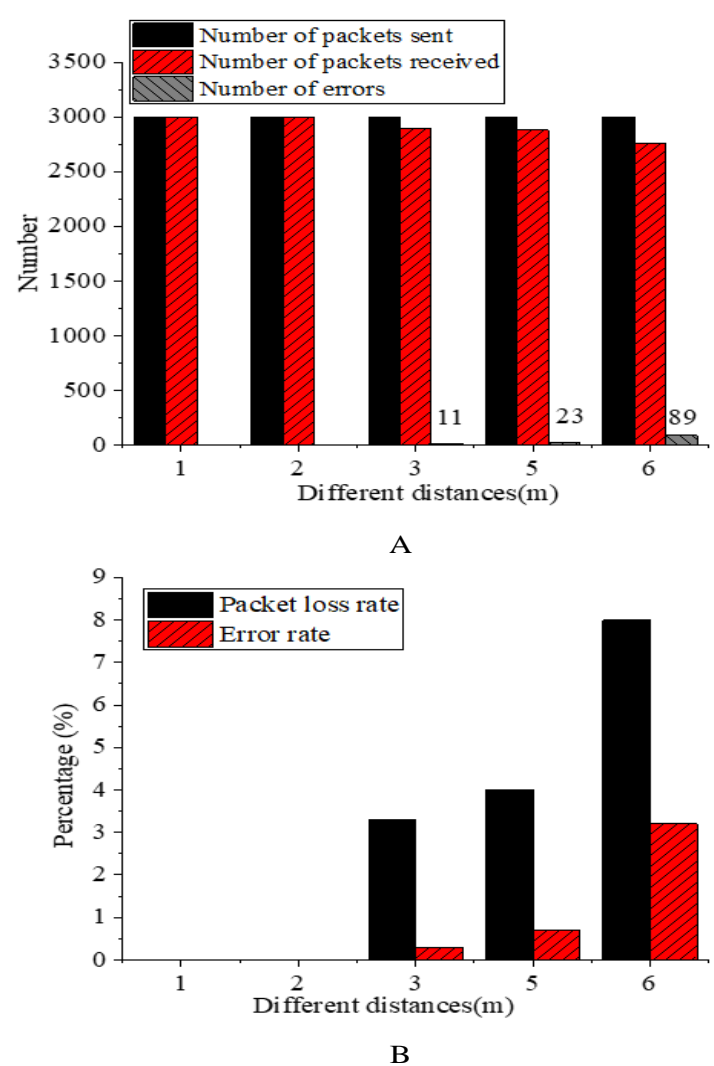

Figure. 8 System test results (A: number of system packets B: data packet acquisition efficiency)

Figure 8 suggests that when the distance between the test content and the node is within two meters, the number of received packets and the number of sent packets are the same, and the number of errors and packet loss rate are both 0 . When the distance between the test content and the node is over two meters, with the increase of distance, the number of packets received gradually reduces, and the packet error rate and packet loss rate increase. This indicates that when the proposed system performs related environmental services, the closer the service content is to the WSN node, the better the service effect is.

\section{Conclusion}

First, the WSN is explained at length, including its concept, features, and functions. Then, the SOA is analyzed theoretically, especially, the typical SOA architecture. Finally, the CC2430 sensor is used as the MCU to propose the WSN service system, and the optimized genetic algorithm is introduced to position the node of the WSN. The results show that the proposed system can display the overall serviceability of the network on the webpage and can display the acquisition results of temperature data. The electrical control webpage of the system can manually close the air conditioner and automatically control the wall lamp. When some nodes are lost due to failure, data can be monitored and transmitted smoothly between the sensors, indicating that if the distance between the adjacent two nodes is within the threshold, the overall communication can be improved of the WSN. When simulating the environmental services, the closer the service content is to the WSN node, the better the service effect is. There are also some shortcomings. First, the Internet system design and service display interface improvement aesthetically Second, the dormancy mechanism and security mechanism should be further analyzed of sensor networks. Third, some service operations are cumbersome and need simplification for a more effective service.

\section{References}

[1] Mao, Guoqiang, Lin, et al. (2016) Efficient Scheduling of Multiple Mobile Chargers for Wireless Sensor Networks. IEEE Transactions on Vehicular Technology, 65(9), 7670-7683.

[2] Boulmaiz A, Messadeg D, Doghmane N, et al. (2016) Robust acoustic bird recognition for habitat monitoring with wireless sensor networks. International Journal of Speech Technology, 19(3), 631-645.

[3] Gidlund M, Han S, Sisinni E, et al. (2018) Guest Editorial From Industrial Wireless Sensor Networks to Industrial Internet of Things. IEEE Transactions on Industrial Informatics, 14(5), 2194-2198.

[4] Lejiang, Guo, Hao, et al. (2017) The Real-Time Detection and Prediction Method for Ballistic Aircraft Based on Distributed Sensor Networks. Wireless Personal Communications, 95(3), 20492072.

[5] Shaoyong, Liang, Bingjia, et al. (2016) Real-Time Detection for Magnetic Island of Neoclassical Tearing Mode in EAST Plasma Control System. Plasma Science and Technology, 18(2), 197-197.

[6] Li-Tao Z, Ling-Ling M, Shou-Shan Z, et al. (2018) Improving the discrimination capability of heavy nuclei based on direct Cherenkov light. Astroparticle Physics, 103, 21-28. 
[7] Hou X, Yin X. (2017) Role of OsWAK124, a rice wall-associated kinase, in response to environmental heavy metal stresses. Pakistan Journal of Botany, 49(4), 1255-1261.

[8] Piatti, Andres E. (2017) Towards a comprehensive knowledge of the open cluster Haffner 9. Monthly notices of the Royal Astronomical Society, 465(3), 2748-2756.

[9] Shafeeq M F, Akbar A H, Zafar S. (2019) SNAG: A Collaborative Routing Architecture for SensorBased Information at SMS Gateway in Cellular Networks. International journal of wireless information networks, 26(4), 367-384.

[10] Rostami A S, Badkoobe M, Mohanna F, et al. (2018) Survey on clustering in heterogeneous and homogeneous wireless sensor networks. Journal of supercomputing, 74(1), 277-323.

[11] Amin R, Biswas G P. (2016) A secure light weight scheme for user authentication and key agreement in multi-gateway based wireless sensor networks[J]. Ad Hoc Networks, 36(part 1), 58-80.

[12] Chen J C, Wen C K, Wong K K. (2016) An Efficient Sensor-Node Selection Algorithm for Sidelobe Control in Collaborative Beamforming. IEEE Transactions on Vehicular Technology, 65(8), 5984-5994.

[13] Afsharinejad A, Davy A, Jennings B. (2016) Dynamic channel allocation in electromagnetic nanonetworks for high resolution monitoring of plants. Nano Communication Networks, 7(mar.), 2-16.

[14] Tresanchez M, Pujol A T, Pallejà, et al. (2018) A proposal of low-cost and low-power embedded wireless image sensor node for IoT applications ScienceDirect. Procedia Computer Science, 134, 99-106.

[15] Gerardo S M, Delamer I M, José L, Martínez L. (2017) A packet scheduler for real-time 6LoWPAN wireless networks in manufacturing systems. Journal of Intelligent Manufacturing, 28(2), 1-11.

[16] Midi D, Bertino E. (2016) Node or Link? FineGrained Analysis of Packet-Loss Attacks in Wireless Sensor Networks. ACM Transactions on Sensor Networks, 12(2), 1-30.
[17] Kyusakov R, Eliasson J, Delsing J, et al. (2016) Integration of Wireless Sensor and Actuator Nodes With IT Infrastructure Using ServiceOriented Architecture. IEEE Transactions on Industrial Informatics, 9(1), 43-51.

[18] Ghouch N E, En-Naimi E M, Kouissi M. (2020) Implementation of an Adaptive Learning System based on Agents and Web Services. International Journal of Emerging Technologies in Learning (iJET), 15(14), 162.

[19] Philip N Y, Rodrigues J J P C, Wang H, et al. (2021) Internet of Things for In-Home Health Monitoring Systems: Current Advances, Challenges and Future Directions. IEEE Journal on Selected Areas in Communications, 39(2), 300-310.

[20] Trepreau J, De Rosny E, Duboc C, et al. (2017) Spectroscopic Characterization of the MetalBinding Sites in the Periplasmic Metal-Sensor Domain of CnrX from Cupriavidus metallidurans CH34. Biochemistry, 50(42), 9036-9045.

[21] Shen Y. (2017) Time Synchronization and Communication Program of Wireless Sensor Network for Online Monitoring of Electrical Equipment. International Journal of Online Engineering, 13(7), 36.

[22] Kyusakov R, Eliasson J, Delsing J, et al. (2016) Integration of Wireless Sensor and Actuator Nodes with IT Infrastructure Using ServiceOriented Architecture. IEEE Transactions on Industrial Informatics, 9(1), 43-51.

[23] Kulkarni V R, Desai V, Kulkarni R V. (2019) A comparative investigation of deterministic and metaheuristic algorithms for node localization in wireless sensor networks. Wireless Networks, 25(5), 2789-2803.

[24] Nuray, At, Hakan, et al. (2018) Multi-hop Efficent Protocol for ZigBee, Bluetooth LowEnergy and ANT Sensor nodes. Journal of Computers, 13(4), 471-479.

[25] Tavakkoli-Moghaddam R, Safari J, Sassani F. (2017) Reliability optimization of series-parallel systems with a choice of redundancy strategies using a genetic algorithm. Reliability Engineering \& System Safety, 93(4), 550-556. 